\title{
A MAXIMUM PRINCIPLE FOR SEMILINEAR PARABOLIC SYSTEMS ${ }^{1}$
}

\author{
ROBERT H. MARTIN, JR.
}

\begin{abstract}
We develop a criterion insuring that every component of the solution to a system of semilinear parabolic equations is strictly positive for positive time. This criterion involves the strict (component-wise) positiveness of solutions to a related ordinary differentiable system.
\end{abstract}

In this note we present a result concerning the strict positiveness of solutions $\vec{u}=\left(u_{1}, \ldots, u_{m}\right)$ to the following system of weakly coupled parabolic equations

$$
\begin{gathered}
\frac{\partial}{\partial t} u_{k}(t, x)=L_{k} u_{k}(t, x)+F_{k}\left(t, x, \vec{u}(t, x), \Delta u_{k}(t, x)\right), \\
t>0, x \in \Omega, y \in \partial \Omega, k=1, \ldots, m, \\
B_{k} u_{k}(t, y)=0, \quad u_{k}(0, x)=\chi_{k}(x) \geqslant 0 .
\end{gathered}
$$

Here $\Omega \subset \mathbf{R}^{n}(n \geqslant 1)$ is a bounded domain with smooth boundary, $\partial \Omega$, and $\Delta$ is the gradient operator (with respect to $x \in \mathbf{R}^{n}$ ). Also, for each $k \in$ $\{1, \ldots, m\}, L_{k}$ is a uniformly elliptic operator with the representation

$$
L_{k} \sim \sum_{i, j=1}^{n} \frac{\partial}{\partial x_{i}}\left(a_{k, i j}(x) \frac{\partial}{\partial x_{j}}\right)
$$

with real-valued, smooth coefficient functions $a_{k, i j}=a_{k, j i}$; and $B_{k}$ is a boundary operator on $(0, \infty) \times \partial \Omega$ of the form

$$
B_{k} u_{k}(t, y)=b_{k}(y) u_{k}(t, y)+\delta_{k} \frac{\partial}{\partial v} u_{k}(t, y)
$$

where $\nu$ is the outward normal on $\partial \Omega$ and either $\delta_{k}=0$ and $b_{k}(y) \equiv 1$ on $\partial \Omega$, or $\delta_{k}=1$ and $b_{k}(y) \geqslant 0$ on $\partial \Omega$. Moreover, the real-valued function $F_{k}$ is $C^{2}$ on $[0, \infty) \times \bar{\Omega} \times \mathbf{R}^{m} \times \mathbf{R}^{n}$ and for each $R>0$ there are numbers $M>0$ and $\gamma \in[0,2)$ such that

$$
\left|F_{k}(t, x, \xi, \eta)\right| \leqslant M\left(1+|\eta|^{\gamma}\right)
$$

whenever $t \in[0, R], x \in \bar{\Omega}, \eta \in \mathbf{R}^{n}$, and $\xi \in \mathbf{R}^{m}$ with $\|\xi\| \leqslant R$.

Received by the editors February 24, 1978 and, in revised form, June 7, 1978.

AMS (MOS) subject classifications (1970). Primary 35K55; Secondary 35K45.

Key words and phrases. Nonlinear parabolic systems, maximum principle, strict positiveness of solutions.

${ }^{1}$ Work supported in part by the U. S. Army Research Office, Research Triangle Park, North Carolina. 
The purpose of this note is to obtain a criterion insuring the strict positiveness of every component for solutions to (PS). Our criterion is based on the behavior of solutions to a related system of ordinary differential equations in $\mathbf{R}^{m}$. Let $\theta$ denote the zero vector in $\mathbf{R}^{m}$ and for each $\xi=\left(\xi_{i}\right)_{1}^{m}$ and $\eta=\left(\eta_{i}\right)_{1}^{m}$ in $\mathbf{R}^{m}$ write $\xi \geqslant \eta$ only in case $\xi_{i} \geqslant \eta_{i}$ for $i=1, \ldots, m$, and write $\xi \gg \eta$ only in case $\xi_{i}>\eta_{i}$ for $i=1, \ldots, m$. Also, let $\theta$ denote the zero of $\mathbf{R}^{n}$. It is assumed that $F=\left(F_{k}\right)_{1}^{m}$ satisfies the following type of quasipositive condition:

$$
\begin{aligned}
& \text { if } k \in\{1, \ldots, m\} \text { and } \xi \in \mathbf{R}^{m} \text { with } \xi \geqslant \theta \text { and } \xi_{k}=0, \\
& \text { then } F_{k}(t, x, \xi, \theta) \geqslant 0 \text { for all }(t, x) \in[0, \infty) \times \Omega \text {. }
\end{aligned}
$$

Throughout this note it is assumed that $x_{0}$ is a (fixed) member of $\Omega$ and that $g=\left(g_{k}\right)_{1}^{m}$ is defined on $[0, \infty) \times \mathbf{R}^{m}$ by

$$
g_{k}(t, \xi)=F_{k}\left(t, x_{0}, \xi, \theta\right) \quad \text { for }(t, \xi) \in[0, \infty) \times \mathbf{R}^{m} \text { and } k=1, \ldots, m \text {. }
$$

Our comparison system of ordinary differential equations is

$$
z^{\prime}(t)=g(t, z(t)), \quad t>0 .
$$

From (1) we see that if $k \in\{1, \ldots, m\}$ and $\xi \geqslant \theta$ with $\xi_{k}=0$, then $g_{k}(t, \xi)$ $\geqslant \theta$, and therefore it is easily deduced that if $z$ is a solution to (ODE) with $z\left(t_{0}\right) \geqslant \theta$, then $z(t) \geqslant \theta$ for all $t \geqslant t_{0}$. Our second main supposition is

$\Gamma$ is a subset of $\{1, \ldots, m\}$ with the property that for each solution $z=\left(z_{i}\right)_{1}^{m}$ to (ODE), the conditions $z\left(t_{0}\right) \geqslant \theta$ and $z_{i}\left(t_{0}\right)>0$ for $i \in \Gamma$ imply $z(t) \gg \theta$ for all $t>t_{0}$.

One should note that if $z\left(t_{0}\right) \gg \theta$, then $z(t) \gg \theta$ for all $t \geqslant t_{0}$, and hence (3) is always satisfied with $\Gamma=\{1, \ldots, m\}$. If each solution $z$ has the property that $z\left(t_{0}\right) \geqslant \theta$ implies $z(t) \gg \theta$ for all $t>t_{0}$, then (3) holds with $\Gamma$ the empty set.

The final assumption is technical, but is less restrictive than requiring $F$ to be quasimonotone:

$$
\begin{aligned}
& \text { if } t \geqslant 0, k \in\{1, \ldots, m\}-\Gamma \text {, and } \xi \geqslant \theta \text { is such that } \xi_{k}=0 \text { and } \\
& F_{k}\left(t, x_{0}, \xi, \theta\right)=0 \text {, then } F_{k}\left(t, x_{0}, \eta, \theta\right)=0 \text { for all } \theta \leqslant \eta \leqslant \xi .
\end{aligned}
$$

Observe that (4) is a consequence of (1) whenever

$$
\partial / \partial \xi_{j} F_{i}\left(t, x_{0}, \xi, \theta\right) \geqslant 0 \text { for } i \neq j \text { (i.e. whenever } F \text { is quasimonotone). }
$$

THEOREM. Suppose $\Gamma$ is a subset of $\{1, \ldots, m\}$ and (1), (3) and (4) are satisfied. Suppose also that the nonnegative initial function $\chi=\left(\chi_{k}\right)_{1}^{m}$ is continuous on $\bar{\Omega}$ and that $\chi_{k}$ is nontrivial for each $k \in \Gamma$. Then there is a $T>0$ such that the solution $\vec{u}=\left(u_{k}\right)_{1}^{m}$ to (PS) exists on $[0, T) \times \Omega$ and satisfies $\dot{u}(t, x) \gg \theta$ for all $(t, x) \in(0, T) \times \Omega$.

For our proof we use the following result:

LEMMA. For each continuous, nonnegative $\chi=\left(\chi_{k}\right)_{1}^{m}$ on $\bar{\Omega}$ there is a $T>0$ such that (PS) has a solution $\vec{u}=\left(u_{k}\right)_{1}^{m}$ on $[0, T) \times \Omega$ with the property that if 
$k \in\{1, \ldots, m\}$, then either $u_{k}(t, x) \equiv 0$ on $(0, T) \times \Omega$ or $u_{k}(t, x)>0$ on $(0, T) \times \Omega$.

We first give the proof of the theorem and then indicate the proof of this lemma.

Proof OF THeOREM. Let $\vec{u}=\left(u_{k}\right)_{1}^{m}$ be the solution to (PS) guaranteed by the Lemma and suppose, for contradiction, that $u_{k}\left(t_{1}, x_{1}\right)=0$ for some $\left(t_{1}, x_{1}\right) \in(0, T) \times \Omega$ and some $k$. Using the Lemma again, we see that if $\Gamma_{0}=\left\{k: u_{k} \equiv 0\right.$ on $\left.(0, T) \times \Omega\right\}$ and $\Gamma_{1}=\left\{k: u_{k}>0\right.$ on $\left.(0, T) \times \Omega\right\}$ then $\Gamma_{0} \neq \varnothing, \Gamma_{0} \cup \Gamma_{1}=\{1, \ldots, m\}, \Gamma_{1} \supset \Gamma$, and $\Gamma_{0} \cap \Gamma_{1}=\varnothing$. It is immediate from (PS) that

$$
F_{k}(t, x, \vec{u}(t, x), \theta) \equiv 0 \quad \text { on }(0, T) \times \Omega \text { and } k \in \Gamma_{0} .
$$

Choose $0<a<b<T$ and select $\xi=\left(\xi_{i}\right)_{1}^{m} \in \mathbf{R}^{m}$ such that $\xi_{i}=0$ for $i \in \Gamma_{0}$ and $0<2 \xi_{i}<u_{i}\left(t, x_{0}\right)$ for $i \in \Gamma_{1}$ and $t \in[a, b]$. From (4) and (5) we have

$$
F_{k}\left(t, x_{0}, \eta, \theta\right)=0 \text { for } t \in[a, b], \theta \leqslant \eta \leqslant 2 \xi, k \in \Gamma_{0} .
$$

Now define the function $z=\left(z_{i}\right)_{1}^{m}$ on $[a, b]$ as follows: $z_{i}(t) \equiv 0$ on $[a, b]$ for $i \in \Gamma_{0}$ and $\left\{z_{i}: i \in \Gamma_{1}\right\}$ satisfies the initial value problem

$$
z_{i}^{\prime}(t)=F_{i}\left(t, x_{0}, z(t), \theta\right), \quad t \in[a, b), z_{i}(a)=\xi_{i}, i \in \Gamma_{1} .
$$

Since $\xi_{i}>0$ for $i \in \Gamma_{1}$, choose $c \in(a, b)$ such that $0<z_{i}(t)<2 \xi_{i}$ for all $t \in[a, c]$ and $i \in \Gamma_{1}$, and then note that

$$
0=F_{i}\left(t, x_{0}, z(t), \theta\right), \quad t \in[a, c), z_{i}(a)=\xi_{i}=0, i \in \Gamma_{0},
$$

by (6). From the definition of $g$ (see (2)) it is immediate from (7) and (7)' that $z$ is a solution to (ODE) on $[a, c)$ with $z_{i}(a)>0$ for $i \in \Gamma_{1} \supset \Gamma$ and $z_{i}(t) \equiv 0$ for $i \in \Gamma_{0}$. This contradicts assumption (3) and we conclude that $\Gamma_{0}$ must be empty. This proves the Theorem once the Lemma is established.

Proof of Lemma. The quasipositive assumption (1) along with a weak form of the maximum principle for parabolic equations implies that the solution $\vec{u}=\left(u_{k}\right)_{1}^{m}$ satisfies $\vec{u}(t, x) \geqslant \theta$ on $[0, T) \times \Omega$ for some $T>0$ (see, e.g., the techniques in Amann [1], Lemmert [4], Lightbourne and Martin [5], and Volkmann [8]). Fix a number $k$ in $\{1, \ldots, m\}$ and for $(t, x) \in[0, T) \times \Omega$, $r \in \mathbf{R}$, and $l \in\{1, \ldots, n\}$ define

$$
w_{k, t, x, r}=\left(\xi_{i}\right)_{1}^{m} \text { where } \xi_{k}=r \text { and } \xi_{i}=u_{i}(t, x) \text { for } i \neq k
$$

and

$$
\begin{aligned}
q_{k, t, x, l, r} & =\left(\eta_{j}\right)_{1}^{n} \quad \text { where } \eta_{l}=r, \eta_{j}=0 \text { for } j<l \text { and } \\
\eta_{j} & =\partial / \partial x_{j} u_{k}(t, x) \text { for } j>l .
\end{aligned}
$$

Now define

$$
\alpha_{k}(t, x)=u_{k}(t, x)^{-1} \int_{0}^{u_{k}(t, x)} \partial / \partial \xi_{k} F_{k}\left(t, x, w_{k, t, x, r}, \Delta u_{k}(t, x)\right) d r
$$


and

$$
\beta_{k, l}(t, x)=h_{l}(t, x)^{-1} \int_{0}^{h_{l}(t, x)} \partial / \partial q_{l} F_{k}\left(t, x, w_{k, t, x, 0}, q_{k, t, x, l, r}\right) d r
$$

where $h_{l}(t, x) \equiv \partial / \partial x_{l} u_{k}(t, x)(l=1, \ldots, n)$. Then

$$
\begin{aligned}
F_{k}\left(t, x, \vec{u}(t, x), \Delta u_{k}(t, x)\right)= & F_{k}\left(t, x, w_{k, t, x, 0}, \theta\right)+\alpha_{k}(t, x) u_{k}(t, x) \\
& +\sum_{l=1}^{m} \beta_{k, l}(t, x) \partial / \partial x_{l} u_{k}(t, x)
\end{aligned}
$$

and since $F_{k}\left(t, x, w_{k, t, x, 0}, \theta\right) \geqslant 0$ by (1) it follows from (PS) that

$$
\partial / \partial t u_{k}(t, x) \geqslant L_{k} u_{k}(t, x)+\alpha_{k}(t, x) u_{k}(t, x)+\sum_{l=1}^{n} \beta_{k, l}(t, x) \partial / \partial x_{l} u_{k}(t, x)
$$

for all $(t, x) \in(0, T) \times \Omega$. Since $u_{k} \geqslant 0$ on $(0, T) \times \Omega$ we have from a strong form of the maximum principle [7, pp. 173 and 175] that $T$ can be chosen so that either $u_{k} \equiv 0$ on $(0, T) \times \Omega$ or $u_{k}>0$ on $(0, T) \times \Omega$. This completes the proof indication of the Lemma.

As an illustration of this result, we consider the mathematical model of a cellular control process with either positive or negative feedback. (See Griffith [2], [3].) This model is the system of three ordinary differential equations

$$
\begin{array}{ll}
z_{1}^{\prime}=-\alpha z_{1}+h\left(z_{3}\right), & z_{1}(0) \geqslant 0, \\
z_{2}^{\prime}=-\beta z_{2}+z_{1}, & z_{2}(0) \geqslant 0, \\
z_{3}^{\prime}=-\gamma z_{3}+z_{2}, & z_{3}(0) \geqslant 0,
\end{array}
$$

where $\alpha, \beta, \gamma$ are positive constants and the function $h$ is defined on $[0, \infty)$ by either $h(r)=r^{\sigma}\left(1+r^{\sigma}\right)^{-1}$ (positive feedback) or $h(r)=\left(1+r^{\sigma}\right)^{-1}$ (negative feedback), and $\sigma \geqslant 1$ is a constant. Defining $F=\left(F_{i}\right)_{1}^{3}$ by the right side of (8):

$$
F_{1}(\xi)=-\alpha \xi_{1}+h\left(\xi_{3}\right) ; \quad F_{2}(\xi)=-\beta z_{2}+z_{1} ; \quad F_{3}(\xi)=-\gamma z_{3}+z_{2} ;
$$

one may easily check that (1), (3) and (4) are satisfied whenever $\Gamma$ is any nonempty subset of $\{1,2,3\}$. From the Theorem we may conclude, for example, if at least one of the nonnegative initial values $\chi_{1}, \chi_{2}$ or $\chi_{3}$ is nontrivial, the solution $\left(u_{i}\right)_{1}^{3}$ to the reaction-diffusion system

$$
\begin{aligned}
\partial / \partial t u_{1} & =d_{1} \Delta u_{1}-\alpha u_{1}+h\left(u_{3}\right), \\
\partial / \partial t u_{2} & =d_{2} \Delta u_{2}-\beta u_{2}+u_{1}, \quad(t, x) \in(0, \infty) \times \Omega, \\
\partial / \partial t u_{3} & =d_{3} \Delta u_{3}-\gamma u_{3}+u_{2}, \\
\left(u_{i}\right)_{1}^{3} & =\left(\chi_{i}\right)_{1}^{3} \text { for } t=0, x \in \Omega, \\
u_{i} & =0 \text { for } t>0, y \in \partial \Omega, \text { and } i=1,2,3,
\end{aligned}
$$

satisfies $u_{i}(t, x)>0$ for all $t>0, x \in \Omega$ and $i=1,2,3$. Here $d_{i}>0$ for $i=1,2,3$ and $\Delta$ is the Laplacian on $\Omega$. Observe that the nonlinearity $F$ is quasimonotone in the case of positive feedback, but not in the case of 
negative feedback. Also, since the results of [6] depend not only on the quasimonotonicity of $F$ but also the irreducibility of the jacobian $F^{\prime}(\theta)$, one sees that the results of [6] establish the strict positiveness of solutions to (9) only in the case of positive feedback with $\sigma=1$.

REMARK. If one assumes that (PS) has a nonnegative solution $\vec{u}$ on $[0, T) \times$ $\Omega$, then the Theorem remains valid under less restrictive assumptions on the smoothness of $F$ and $\partial \Omega$. Note that it is necessary only to be assured that each component of $\vec{u}$ is either strictly positive or identically zero for the Theorem to hold.

\section{REFERENCES}

1. H. Amann, Invariant sets and existence theorems for semi-linear parabolic and elliptic systems (to appear).

2. J. S. Griffith, Mathematics of cellular control processes. I. Negative feedback to one gene, J. Theoret. Biol. 20 (1968), 202-208.

3. Mathematics of cellular control processes. II. Positive feedback to one gene, J. Theoret. Biol. 20 (1968), 209-216.

4. R. Lemmert, Uber die Invarianz einer konvexen Menge in bezug auf Systeme von gewöhnlichen, parabolischen und elliptischen Differentialgleichungen, Math. Ann. 230 (1977), 49-56.

5. J. H. Lightbourne and R. H. Martin, Relatively continuous nonlinear perturbations of analytic semigroups, JNA-TMA 1 (1977), 277-292.

6. R. H. Martin, Jr., Asymptotic stability and critical points for nonlinear quasimonotone parabolic systems, J. Differential Equations (to appear).

7. M. H. Protter and H. F. Weinberger, Maximum principles in differential equations, PrenticeHall, Englewood Cliffs, N. J., 1967.

8. P. Volkmann, Über die positive Invarianz einer abgeschlossenen Teilmenge eines Banachschen Raumes bezüzlich der Differentialgleichung $u^{\prime}=f(t, u)$, J. Reine Angew. Math. 285 (1976), 59-65.

Department of Mathematics, North Carolina State University, Raleigh, North Carolina 27607 